\title{
Comparison of Different Approaches in Lumbosacral Spinal Fusion Surgery: A Systematic Review and Meta-Analysis
}

\author{
Maximilian Lenz, Kaliye Mohamud, Jan Bredow, Stavros Oikonomidis, Peer Eysel, Max Joseph Scheyerer \\ Department of Orthopaedic and Trauma Surgery, University Hospital of Cologne, Cologne, Germany
}

We aimed to systematically review the literature to analyze the differences in posterior lumbar interbody fusion (PLIF), anterior lumbar interbody fusion (ALIF), and transforaminal lumbar interbody fusion (TLIF), focusing on the complications, risk factors, and fusion rate of each approach. Spinal fusion surgery is a well-established surgical procedure for a variety of indications, and different approaches developed. The various approaches and their advantages, as well as approach-related pathology and complications, are well investigated in spinal surgery. Focusing only on lumbosacral fusion, the comparative studies of different approaches remain fewer in numbers. We systematically reviewed the literature on the complications associated with lumbosacral interbody fusion. Only the PLIF, ALIF, or TLIF approaches and studies published within the last decade (2007-2017) were included. The exclusion criteria in this study were oblique lumbar interbody fusion, extreme lateral interbody fusion, more than one procedure per patient, and reported patient numbers less than 10. The outcome variables were indications, fusion rates, operation time, perioperative complications, and clinical outcome by means of Visual Analog Scale, Oswestry Disability Index, and Japanese Orthopaedic Association score. Five prospective, 17 retrospective, and two comparative studies that investigated the lumbosacral region were included. Mean fusion rates were 91,4\%. ALIF showed a higher operation time, while PLIF resulted in greater blood loss. In all approaches, significant improvements in the clinical outcome were achieved, with ALIF showing slightly better results. Regarding complications, the ALIF technique showed the highest complication rates. Lumbosacral fusion surgery is a treatment to provide good results either through an approach for various indications as causes of lower back pain. For each surgical approach, advantages can be depicted. However, perioperative complications and risk factors are numerous and vary with ALIF, PLIF, and TLIF procedures, as well as with fusion rates.

Keywords: L5/S1 fusion; Risk factors; Complications; Posterior lumbar interbody fusion; Transforaminal lumbar interbody fusion; Anterior lumbar interbody fusion

\section{Introduction}

Spinal fusion surgery has a long history in the treatment of different causes of lower back pain. In the past, especially in the last 4 decades, the number of these surgeries has significantly increased. Nowadays, spinal fusion surgery provides a primary treatment for various conditions responsible for degenerative forms of lower back pain [1]. Over time, the so-called $360^{\circ}$ fusion surgery was achieved by all surgical approaches, with posterior lumbar interbody fusion (PLIF) being the first to be carried out in 1947. Since then, spinal fusion has evolved to include

Received Aug 12, 2020; Revised Sep 10, 2020; Accepted Sep 25, 2020

Corresponding author: Maximilian Lenz

Department of Orthopaedic and Trauma Surgery, University Hospital of Cologne, Kerpener Str. 62, D-50937 Cologne, Germany

Tel: +49-221-478-86252, Fax: +49-221-478-87296, E-mail: maximilian.lenz@uk-koeln.de 
anterior, transforaminal, extreme, lateral, and axial approaches [2].

According to several studies, anterior lumbar interbody fusion (ALIF) procedures have increased in popularity in recent years compared to the transforaminal lumbar interbody fusion (TLIF) and PLIF approaches. Possible reasons may include better access to disc space, direct visualization of the anterior column, and a wide surface to allow insertion of big surgical grafts $[3,4]$. However, the retroperitoneal approach consequently has a higher risk of vascular and ureteral injury. The PLIF approach, which has developed over the years, reducing prevalent complications, is a widely-accepted procedure using pedicle screws and interbody cages, which can be either polyetheretherketone or carbon fiber polymer cages as well as titanium cages [5]. PLIF uses a traditional lumbar approach which most spinal surgeons are familiar with; thus, they are more comfortable using this method [2]. TLIF as an alternative posterior approach to the interbody space was described, with its advantage of being associated with significantly fewer perioperative complications [3]. It is also associated with a decrease in morbidity, greater stability, and larger increase in segmental lordosis resulting in better outcomes [6]. For lumbar interbody fusion, surgery risks and complications including wound infection, bleeding, nerve damage and recurrent pain are prominent. Furthermore, pedicle screw loosening and non-union are common postoperative complications [7]. Allograft or cage dislocation might also lead to revision surgery [8]. Due to these complications, the failure rate of lumbar interbody fusion has been reported to be as high as $37 \%$ among patients in the United States $[9,10]$.

The lumbosacral segment takes a special position here, as it is located between the mobile lumbar spine and the immobile sacrum which is fixed between the two ilia [11]. Due to little evidence regarding the best approach, the choice is commonly based upon surgeons' preferences and not the pathology in question, location of the affected segmented, or number of segments to be fused [2]. The lumbosacral segment is a challenging anatomical region for screw and cage positioning [12-14]. To the best of our knowledge, no studies comparing the different complications and risk factors in lumbosacral fusion surgery with the various indications for each approach have been published. Therefore, this study aimed to provide an overview of the complications and risk factors associated with PLIF, ALIF, and TLIF in lumbosacral fusion.

\section{Methods}

\section{Inclusion criteria}

We systematically reviewed the available literature covering lumbosacral interbody fusion and associated risk factors and complications. This systematic review was conducted according to the PRISMA (Preferred Reporting Items for Systematic Reviews and Meta-Analyses) guidelines (Fig. 1) [15,16]. Between June and August 2017, randomized controlled studies and observational studies including retrospective and prospective studies were searched via PubMed, Science Direct, Wiley Online Library, and Ovid Medline databases. The keywords "PLIF," "posterior lumbar interbody fusion," "PLIF risk factors and complications," "ALIF," "anterior lumbar interbody fusion," "ALIF risk factors and complications," "TLIF," "transforaminal lumbar interbody fusion," “TLIF risk factors and complications," "L5/S1 Fusion," and "non-union L5/S1" were searched for analysis. The search was limited

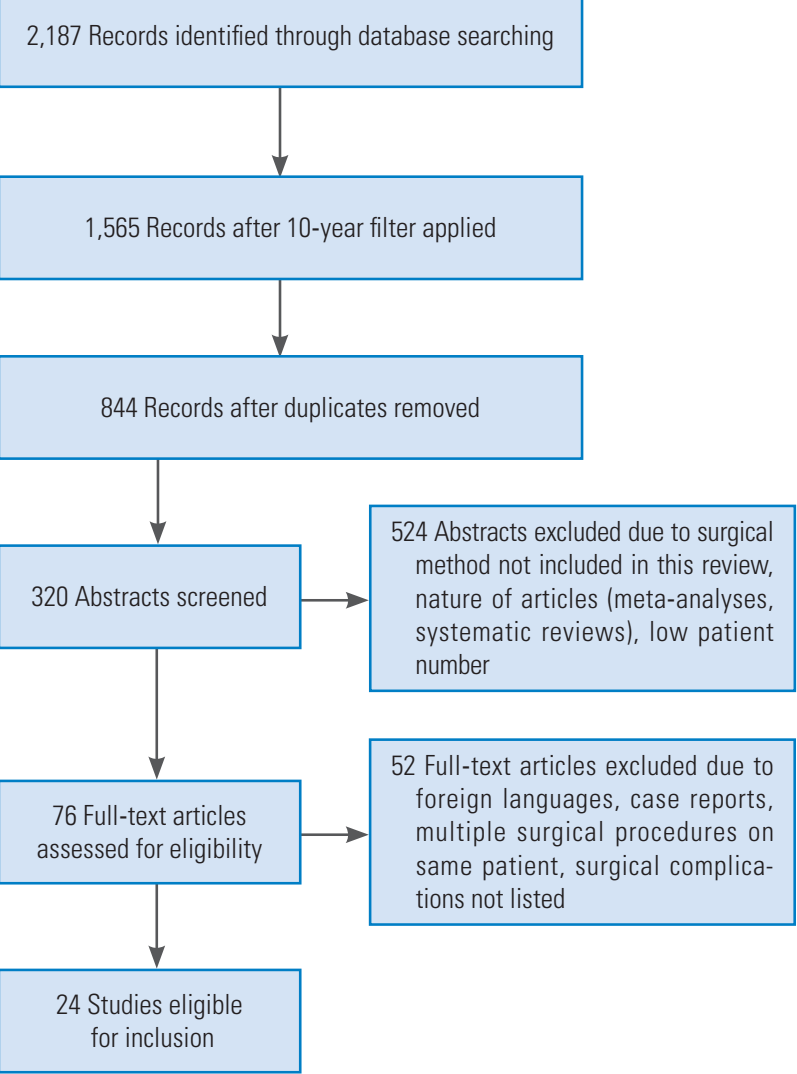

Fig. 1. Outline of the literature research according to the PRISMA (Preferred Reporting Items for Systematic Reviews and Meta-Analyses) guidelines. 
to articles published in the last decade (2007 to 2017) and only full texts written in either English or German were selected. Titles and abstracts of potentially related articles identified by the electronic search were reviewed. Indications for fusion surgery at the lumbosacral level were neither inclusion nor exclusion criteria. Given that this study was conducted according to ethical guidelines, no further institutional approval was needed.

\section{Exclusion criteria}

As we only included anterior (ALIF) and posterior (PLIF/ TLIF) lumbar approaches, we did not include any studies that focused on either the lateral lumbar interbody fusion (LLIF), extreme lateral interbody fusion (XLIF), or oblique lumbar interbody fusion (OLIF) technique, more than one procedure on the same patient, publications older than 2007, and studies that reported less than 10 patients. LLIF is a lateral approach passing through the retroperitoneal fat and psoas muscle with XLIF being a modification in LLIF as a minimally-invasive option using a special position of the patient. From the lateral approach, typically, the L5/S1 segment cannot be accessed. When studies compared two approaches with one of them being either LLIF/XLIF or OLIF, only ALIF/TLIF and PLIF patient data were used for analysis. Meta-analyses, systematic reviews, and case reports were not included in the evaluation.

\section{Study selection}

We included all randomized controlled and observational studies, including retrospective and prospective studies. To include and exclude studies according to the defined criteria, one reviewer searched titles and abstracts of all identified publications, and, when necessary, the full text of each article along with its references. The Visual Analog Scale (VAS), Oswestry Disability Index (ODI), and Japanese Orthopaedic Association score (JOA score) were used for clinical outcome measures.

\section{Statistical analysis}

Statistical analysis was performed using Microsoft Excel (Microsoft Corp., Redmond, WA, USA) for all identified and included studies. Data extraction was performed to calculate mean scores when data were published.

\section{Results}

We included 24 studies for evaluation; 17 retrospective, five prospective, and two comparative studies. We included six studies on ALIF alone, eight on PLIF, and two on TLIF alone. Two studies compared PLIF and TLIF, two studies compared PLIF and ALIF, one study compared ALIF and LLIF, two studies compared ALIF and TLIF, and one study compared PLIF, PLF, and TLIF. With the exception of one, all studies included the fusion of the L5/ S1 segment in the patient cohort. A total of 2,846 patients were included in this systematic review and meta-analysis. Follow-up data were retrieved from the studies as shown

Table 1. Follow-up data in months regarding minimum follow-up, mean followup, and range in months of follow-up

\begin{tabular}{|c|c|c|c|}
\hline Author & $\begin{array}{l}\text { Min follow- } \\
\text { up (mo) }\end{array}$ & Mean follow-up (mo) & $\begin{array}{l}\text { Range } \\
\text { (mo) }\end{array}$ \\
\hline Hsieh et al. [22] (2017) & 12 & 22.36 & $12-37$ \\
\hline Mobbs et al. [4] (2016) & 10 & 15 & NA \\
\hline Lee et al. [23] (2015) & 12 & NA & NA \\
\hline Pinson et al. [24] (2017) & 36 & NA & NA \\
\hline De Kunder et al. [20] (2017) & 2 & NA & $2-12$ \\
\hline Hayashi et al. [25] (2016) & 24 & 40.45 & $24-74$ \\
\hline Rothenfluh et al. [17] (2014) & NA & NA & NA \\
\hline Kim et al. [26] (2010) & 24 & NA & NA \\
\hline Okuda et al. [27] (2014) & 24 & 96 & $24-204$ \\
\hline Gologorsky et al. [28] (2014) & 37 & 52 & $37-63$ \\
\hline Sakaura et al. [29] (2016) & 24 & 35.4 & $25-41$ \\
\hline Dorward et al. [3] (2013) & 24 & 33 (TLIF), 52 (ALIF) & NA \\
\hline Makino et al. [18] (2017) & 24 & 24 & NA \\
\hline Fleege et al. [30] (2016) & NA & 94.8 (ALIF), 67.2 (PLIF) & NA \\
\hline Baeesa et al. [31] (2016) & NA & 219 & $243-267$ \\
\hline Lara-Almunia et al. [21] (2015) & 12 & NA & NA \\
\hline Malham et al. [32] (2016) & NA & 34.1 & $24-60$ \\
\hline Audat et al. [6] (2012) & 3 & NA & $3-36$ \\
\hline Hosono et al. [33] (2008) & 12 & 75.8 & $12-110$ \\
\hline Min et al. [34] (2007) & 24 & 44.6 & $24-68$ \\
\hline Quraishi et al. [35] (2013) & NA & NA & NA \\
\hline Rao et al. [36] (2015) & NA & 20 & $12-42$ \\
\hline Li et al. [37] (2017) & NA & 4.3 & $2-7$ \\
\hline Jang et al. [19] (2015) & 3 & NA & NA \\
\hline Mean of all & 19 & 53.9 & $2-267$ \\
\hline
\end{tabular}

NA, not available; TLIF, transforaminal lumbar interbody fusion; ALIF, anterior lumbar interbody fusion; PLIF, posterior lumbar interbody fusion. 
Table 2. Overview of indications with ALIF, PLIF, and TLIF approach

\begin{tabular}{|c|c|c|c|c|c|c|}
\hline \multirow{2}{*}{ Indication for surgery } & \multicolumn{2}{|c|}{ ALIF } & \multicolumn{2}{|c|}{ PLIF } & \multicolumn{2}{|c|}{ TLIF } \\
\hline & No. of studies & No. of patients & No. of studies & No. of patients & No. of studies & No. of patients \\
\hline Degenerative disc disease & 3 & 465 & 2 & 72 & 1 & 37 \\
\hline Degenerative spondylolisthesis & 3 & 40 & 5 & 173 & 3 & 202 \\
\hline Isthmic spondylolisthesis & 4 & 114 & 6 & 240 & 1 & 15 \\
\hline General spondylolisthesis & 3 & 76 & 2 & 289 & & \\
\hline Scoliosis & 3 & 42 & 1 & 31 & & \\
\hline Spinal stenosis & & & 2 & 35 & 1 & 398 \\
\hline Disc herniation & & & & & 1 & 54 \\
\hline
\end{tabular}

ALIF, anterior lumbar interbody fusion; PLIF, posterior lumbar interbody fusion; TLIF, transforaminal lumbar interbody fusion.

in Table $1[3,4,6,17-37]$. The mean follow-up time for all studies was 53.9 months (nine studies presented no mean follow-up data) with 19 months being the mean minimum follow-up time (range, 2 to 267 months; eight studies presented no minimum follow-up data).

The indications for surgery were relatively similar across the studies (Table 2). Most ALIF patients had degenerative disc disease, isthmic spondylolisthesis, and scoliosis. The main indications for PLIF were spondylolisthesis (isthmic and general), degenerative disc disease, and spinal stenosis. The TLIF patients' most common surgical indications were spinal stenosis, degenerative spondylolisthesis, and disc herniation. Only four studies did not present surgical indications.

In the aspect of fusion rates, ALIF reached the highest rates with $97.8 \%$ (range, $94.4 \%$ to $100 \%$ ) followed by TLIF with $96 \%$ (range, $91.9 \%$ to $100 \%$ ) (Table 3). PLIF showed a fusion rate of $91.4 \%$ (range, $84 \%$ to $100 \%$ ) (Table 2). PLIF included nine studies with 605 patients, ALIF included six studies with 559 patients, while TLIF only was analyzed in two studies with 79 patients.

Thirteen studies reported the intraoperative blood loss as well as the mean duration of surgery, while three studies reported only one of the two parameters. The ALIF group had longer surgeries $(\mathrm{n}=6)$. PLIF $(\mathrm{n}=7)$ and TLIF $(n=2)$ groups had similar surgery durations (Table 4$)$. Regarding the blood loss, the ALIF approach $(n=5)$ showed a clear advantage, with an almost $50 \%$ reduction in blood loss compared to the PLIF $(n=6)$ and TLIF $(n=2)$.

All included studies reported clinical outcome parameters to assess postoperative clinical results. In our systematic review, we analyzed ODI, VAS, and JOA scores, which are commonly used. Four studies did not provide data
Table 3. Fusion rates

\begin{tabular}{lcc} 
Fusion method & No. of studies & Fusion rate $(\%)$ \\
\hline ALIF & 6 & $97.8 \pm 2.61(94.4-100.0)$ \\
PLIF & 9 & $91.4 \pm 4.51(84.0-100.0)$ \\
\hline TLIF & 2 & $96.0 \pm 5.72(91.9-100.0)$ \\
\hline
\end{tabular}

Values are presented as number or mean \pm standard deviation (range).

ALIF, anterior lumbar interbody fusion; PLIF, posterior lumbar interbody fusion; TLIF, transforaminal lumbar interbody fusion.

Table 4. Mean operating times and blood loss

\begin{tabular}{lcc} 
Fusion method & Operating time $(\min )$ & Blood loss $(\mathrm{mL})$ \\
\hline ALIF $(n=6)$ & $198.4 \pm 81.5$ & $278.5 \pm 144.4$ \\
$\operatorname{PLIF}(n=7)$ & $173.3 \pm 64.3$ & $440.2 \pm 198.8$ \\
$\operatorname{TLIF}(n=2)$ & $173.2 \pm 40.0$ & $467 \pm 25.5$ \\
\hline
\end{tabular}

Values are presented as mean \pm standard deviation.

ALIF, anterior lumbar interbody fusion; PLIF, posterior lumbar interbody fusion; TLIF, transforaminal lumbar interbody fusion.

of any on our chosen scores, and for TLIF, no JOA data could be reported. Regarding VAS and ODI, there was no significant difference in postoperative outcomes between all techniques (Tables 5-7 for detailed results).

Regarding perioperative complications, the average complication rates were $16.1 \%$ for the 11 PLIF studies, $18.7 \%$ for the 11 ALIF studies, and $13.3 \%$ for the seven TLIF studies (Table 7). The most common complications in the ALIF group were vascular injury, wound infection, and ileus, which occurred at a rate of $3.6 \%, 2.7 \%$, and $2.5 \%$, respectively. The PLIF group showed neurological deficit, dural leakage, implant failure, and adjacent segment disease (ASD) to be the most common complications with occurrence rates of $7.3 \%, 3.7 \%, 3.3 \%$, and $3.1 \%$, re- 
Table 5. Visual Analog Scale values

\begin{tabular}{lrrr} 
Fusion method & Preoperative & Postoperative & Difference \\
\hline ALIF & $7.4 \pm 0.6$ & $2.5 \pm 0.74$ & $5.0 \pm 0.86$ \\
PLIF & $7.6 \pm 0.38$ & $2.4 \pm 0.71$ & $5.5 \pm 0.71$ \\
TLIF & $6.5 \pm 0.81$ & $2.1 \pm 0.53$ & $4.3 \pm 0.26$ \\
\hline
\end{tabular}

Values are presented as mean \pm standard deviation.

ALIF, anterior lumbar interbody fusion; PLIF, posterior lumbar interbody fusion; TLIF, transforaminal lumbar interbody fusion.

Table 6. Oswestry Disability Index values

\begin{tabular}{lccc} 
Fusion method & Preoperative & Postoperative & Difference \\
\hline ALIF & $54.8 \pm 4.28$ & $21.2 \pm 5.8$ & $32.0 \pm 8.48$ \\
\hline PLIF & $52.2 \pm 17.62$ & $15.3 \pm 7.71$ & $31.1 \pm 14.66$ \\
\hline TLIF & $53.8 \pm 5.31$ & $14.6 \pm 3.18$ & $39.2 \pm 2.21$ \\
\hline
\end{tabular}

Values are presented as mean \pm standard deviation.

ALIF, anterior lumbar interbody fusion; PLIF, posterior lumbar interbody fusion; TLIF, transforaminal lumbar interbody fusion.

Table 7. Japanese Orthopaedic Association value

\begin{tabular}{lccc} 
Fusion method & Preoperative & Postoperative & Difference \\
\hline ALIF & $12.4 \pm 2.52$ & $20.87 \pm 4.86$ & $8.51 \pm 2.67$ \\
PLIF & $9.3 \pm 1.5$ & $13.6 \pm 0.8$ & 4.3 \\
\hline
\end{tabular}

Values are presented as mean \pm standard deviation.

ALIF, anterior lumbar interbody fusion; PLIF, posterior lumbar interbody fusion.

spectively. The TLIF group reported neurological deficit, hardware failure, and non-union as the most common complications, occurring rates of $4.1 \%, 1.2 \%$, and $1.1 \%$, respectively (Table 8).

Seven studies reported risk factors associated with the fusion method used. For ALIF, higher vascular complications occurred in patients with vascular and cardiac risk factors such as atherosclerosis, calcification, and osteophytes on lumbar spine X-rays [17]. Higher blood loss due to venous injuries was observed more frequently than caused by arterial injuries in PLIF and TLIF. In PLIF, old age and minor lumbar lordosis changes were risk factors for pain-related disorders and psychological and social life dysfunction [18]. Non-union was observed in more patients when lumbar spine dysfunction was present, whereas another study reported only the operation time as a significant risk factor for the occurrence of complications $(p>0.05)$. For TLIF, preoperative segmental angle and change after surgery at index level was reported as a significant risk factor for contralateral radiculopathy after unilateral TLIF [19].
Table 8. Intra and postoperative complications for each approach

\begin{tabular}{lrrr}
\hline Complications & ALIF & PLIF & TLIF \\
\hline Vascular injury & 24 & 0 & 0 \\
\hline Dural leakage & 0 & 37 & 5 \\
\hline Peritoneal opening & 12 & 0 & 0 \\
\hline Wound infection & 18 & 16 & 2 \\
\hline Non-union & 5 & 18 & 10 \\
\hline Implant failure & 15 & 33 & 11 \\
\hline Cage migration & 0 & 10 & 10 \\
\hline Neurological deficits & 12 & 73 & 38 \\
\hline Adjacent segment disease & 11 & 31 & 0 \\
\hline Ileus & 17 & 0 & 4 \\
\hline Deep vein thrombosis/pulmonary embolism & 5 & 2 & 0 \\
\hline Retrograde ejaculation & 16 & 0 & 0 \\
\hline Incisional hernia & 5 & 0 & 0 \\
\hline Pneumonia & 2 & 1 & 4 \\
\hline Hematoma & 4 & 6 & 0 \\
\hline Stroke & 0 & 1 & 0 \\
\hline
\end{tabular}

ALIF, anterior lumbar interbody fusion; PLIF, posterior lumbar interbody fusion; TLIF, transforaminal lumbar interbody fusion.

\section{Discussion}

Previous studies focused on comparisons between various surgical techniques and discussed their respective advantages and disadvantages. Consequently, this study focused on the complications and risk factors to add a different perspective to previously published research. A metaanalysis conducted in 2017 declared ALIF to be the most researched surgical approach, followed by PLIF and TLIF [38].

This meta-analysis covered the complications and risk factors surrounding lumbosacral interbody fusion surgeries. The indications for the different surgical approaches in lumbosacral fusion are relatively similar, with spondylolisthesis being the most common across the three approaches.

At the lumbosacral junction, ALIF seems to be the method of choice when treating discogenic back pain or reconstructing a lordosis [39]. Generally, the rates of perioperative mortality and complications are higher with the ALIF approach, which is likely due to the proximity to vital organs when accessing the abdominal and thoracic cavities [40]. In our findings, these specific complications included peritoneal opening, incisional hernia, vascular injury, and retrograde ejaculation. Previous studies showed rates of vascular injury ranging from $1.9 \%$ to $24 \%$, 
emphasizing the fact that our rate of $3.6 \%$ was within the range. The rate of retrograde ejaculation was $2.4 \%$, and has been reported to occur in up to $5 \%$ of patients [41]. The rate of incisional hernia, which is a rare complication of ALIF, was 0.7\% [42]. An interesting observation was that the ALIF studies reported 24 cases of intraoperative vascular injuries, whereas PLIF and TLIF did not have a single case of vascular injury in the selected studies, but still reported the least mean blood loss. Although previous studies reported ALIF as having the highest rate of cage migration, in this study, the ALIF trials did not record any case of cage migration, while the PLIF and TLIF trials had 10 cases each [38]. The rates of non-union for ALIF, PLIF, and TLIF were $0.7 \%, 1.8 \%$, and $1.1 \%$, respectively. Accordingly, ALIF had the highest rate of fusion followed by TLIF, and lastly, PLIF. In summary, ALIF is a challenging technique due to its anterior approach with the occurrence of specific complications, but achieves the highest fusion rates.

The findings of this study showed that TLIF had the lowest complication rate compared to ALIF and PLIF, which is consistent with the findings of previous studies [43]. This might be because TLIF is the least invasive approach and with the unilateral access to the foraminal space, many potential complications such as nerve root damage and dural tear can be avoided [2]. The posterior approaches of PLIF and TLIF lead to higher risks of nerve root damage, which results in complications such as neurological deficit and dural tear. However, for TLIF, a 50\% lower complication rate was reported compared to PLIF [20]. This is possibly due to the less-invasive nature of TLIF, and thus sparing the lamina, facet, and pars on the contralateral side in the unilateral approach, which reduces the chance of damage to these sensitive structures [44]. ASD is a common complication that occurs after lumbar fusion surgery, independently of the approach used in the longer term of follow-up. The time lapse until ASD becomes critical is a matter of years rather than months. In the included studies that analyzed TLIF, the follow-up period was shorter, with a mean follow-up of 34.8 months (minimum, 17 months; range, 3 to 63 months), while the mean follow-up for ALIF and PLIF was greater mean 52.6 months (minimum, 19 months; range, 2 to 267 months). In terms of biomechanical differences between the approaches, the unilateral approach in TLIF assumes advantages hypothesizing a lower rate of ASD as less vertebral bone is resected for cage insertion when compared to
PLIF. Compared to ALIF, the higher fusion rate in ALIF might lead to a more rigid fixation allowing increase in stress load in adjacent segments. However, as the followup is not comparable, and TLIF studies reveal no data regarding ASD, only a limited amount of prediction regarding ASD in TLIF can be made.

Taking into account the clinical outcome, TLIF had the shortest surgery time and is the least invasive approach; however, the highest blood loss was observed with this approach. ALIF had the longest surgery time and showed minimal blood loss. Regarding pain scores, PLIF had the greatest improvement in VAS and the least improvement in the ODI score. TLIF had the least improvement in VAS along with the greatest improvement in the ODI score. Other comparative studies have also shown that TLIF is better than the other approaches in the ODI score improvement post-surgery [38]. All approaches had a mean postoperative VAS score in the "mild pain" range, PLIF and TLIF scored mean postoperative ODI scores in the "minimal disability" range, and ALIF in the "moderate disability" range.

Previous studies have shown that mortality was highest in patients with advanced age (above 75 years) [40]. However, Lara-Almunia et al. [21] contradicted this finding by concluding that age, among other factors, did not significantly increase the risk of post-surgical complications. The ages of the 36 patients in their study ranged from 30 to 82 years, with a mean value of 57.1 years. This has been supported by other studies in the past which have also shown that advanced age does not necessarily increase the mortality rate [21].

In line with previous findings, the reported risk factors of PLIF such as fixation of multiple levels, longer operations, and non-union have shown a positive link between these factors and complication rates [45]. Continuous bleeding from the operation site while proceeding at other levels was one reason for the increased rate of complications associated with longer surgeries [45]. In aspects of cage placement, smaller cages increase the likelihood of posterior cage migration, which was also corroborated by previous findings. Furthermore, smaller cages show reduced contact surface area, and therefore increase stress on the endplates, resulting in higher rates of ASD [46]. Small contralateral lumbar foraminal dimensions cause compression of the nerve roots that are associated with contralateral radiculopathy in the unilateral approach in TLIF [19]. Furthermore, vascular risk factors, including 
diabetes or calcified vessels predisposing to plaque rupture, significantly increase the rate of vascular complications in the ALIF groups [17].

One main limitation was the inadequate availability of randomized prospective trials for lumbosacral spinal fusion surgery. Hence, there were only five prospective trials against 19 retrospective ones. Therefore, a higher publication bias must be considered, and may influence the validity of the results. Furthermore, typically, in a systematic review, the risk of selection bias due to inclusion and exclusion criteria is also present. Moreover, the heterogeneity of the surgical indications also poses a limitation, as certain conditions are more prone to complications than others. The usual problem of low patient numbers used in the trials has also affected our study. Moreover, some studies either only provided limited patient data or did not clearly define complications and report surgical parameters. Finally, the surgical differences such as fusion levels, implant material, surgeon's experience, and especially the unknown reason to choose either approach should also be considered as limitations of our study.

\section{Conclusions}

To conclude, our findings showed the strengths and weaknesses of the different approaches, with complications and risk factors highlighted for each one. Similarities between them included the indications for surgery, mean surgery times, and the mean fusion rates which were all above $90 \%$ for all the approaches. However, ALIF showed the highest fusion rates, and TLIF had the fewest complications in lumbosacral fusion surgery.

\section{Conflict of Interest}

No potential conflict of interest relevant to this article was reported.

\section{References}

1. Rajaee SS, Bae HW, Kanim LE, Delamarter RB. Spinal fusion in the United States: analysis of trends from 1998 to 2008. Spine (Phila Pa 1976) 2012;37:6776.

2. Mobbs RJ, Phan K, Malham G, Seex K, Rao PJ. Lumbar interbody fusion: techniques, indications and comparison of interbody fusion options including
PLIF, TLIF, MI-TLIF, OLIF/ATP, LLIF and ALIF. J Spine Surg 2015;1:2-18.

3. Dorward IG, Lenke LG, Bridwell KH, et al. Transforaminal versus anterior lumbar interbody fusion in long deformity constructs: a matched cohort analysis. Spine (Phila Pa 1976) 2013;38:E755-62.

4. Mobbs RJ, Phan K, Assem Y, Pelletier M, Walsh WR. Combination Ti/PEEK ALIF cage for anterior lumbar interbody fusion: early clinical and radiological results. J Clin Neurosci 2016;34:94-9.

5. Li CS, Vannabouathong C, Sprague S, Bhandari M. The use of carbon-fiber-reinforced (CFR) peek material in orthopedic implants: a systematic review. Clin Med Insights Arthritis Musculoskelet Disord 2015;8:33-45.

6. Audat Z, Moutasem O, Yousef K, Mohammad B. Comparison of clinical and radiological results of posterolateral fusion, posterior lumbar interbody fusion and transforaminal lumbar interbody fusion techniques in the treatment of degenerative lumbar spine. Singapore Med J 2012;53:183-7.

7. Kim YJ, Bridwell KH, Lenke LG, Rhim S, Cheh G. Pseudarthrosis in long adult spinal deformity instrumentation and fusion to the sacrum: prevalence and risk factor analysis of 144 cases. Spine (Phila Pa 1976) 2006;31:2329-36.

8. Sherman J. Spinal fusion risks and complications. Deerfield (IL): Veritas Health; 2006.

9. Eichholz KM, Ryken TC. Complications of revision spinal surgery. Neurosurg Focus 2003;15:E1.

10. Tsuchiya K, Bridwell KH, Kuklo TR, Lenke LG, Baldus C. Minimum 5-year analysis of L5-S1 fusion using sacropelvic fixation (bilateral S1 and iliac screws) for spinal deformity. Spine (Phila Pa 1976) 2006;31:303-8.

11. Tropiano P, Giorgi H, Faure A, Blondel B. Surgical techniques for lumbo-sacral fusion. Orthop Traumatol Surg Res 2017;103:S151-9.

12. Yoshii T, Hirai T, Yamada T, et al. Lumbosacral pedicle screw placement using a fluoroscopic pedicle axis view and a cannulated tapping device. J Orthop Surg Res 2015;10:79.

13. Bredow J, Boese CK, Werner CM, et al. Predictive validity of preoperative CT scans and the risk of pedicle screw loosening in spinal surgery. Arch Orthop Trauma Surg 2016;136:1063-7.

14. Lehman RA Jr, Kuklo TR, Belmont PJ Jr, Andersen 
RC, Polly DW Jr. Advantage of pedicle screw fixation directed into the apex of the sacral promontory over bicortical fixation: a biomechanical analysis. Spine (Phila Pa 1976) 2002;27:806-11.

15. Moher D, Liberati A, Tetzlaff J, Altman DG; PRISMA Group. Preferred reporting items for systematic reviews and meta-analyses: the PRISMA statement. Int J Surg 2010;8:336-41.

16. Page MJ, Moher D. Evaluations of the uptake and impact of the preferred reporting items for systematic reviews and meta-analyses (PRISMA) statement and extensions: a scoping review. Syst Rev 2017;6:263.

17. Rothenfluh DA, Koenig M, Stokes OM, Behrbalk E, Boszczyk BM. Access-related complications in anterior lumbar surgery in patients over 60 years of age. Eur Spine J 2014;23 Suppl 1:S86-92.

18. Makino T, Kaito T, Fujiwara H, et al. Risk factors for poor patient-reported quality of life outcomes after posterior lumbar interbody fusion: an analysis of 2-year follow-up. Spine (Phila Pa 1976) 2017;42:150210.

19. Jang KM, Park SW, Kim YB, Park YS, Nam TK, Lee YS. Acute contralateral radiculopathy after unilateral transforaminal lumbar interbody fusion. J Korean Neurosurg Soc 2015;58:350-6.

20. De Kunder SL, van Kuijk SMJ, Rijkers K, et al. Transforaminal lumbar interbody fusion (TLIF) versus posterior lumbar interbody fusion (PLIF) in lumbar spondylolisthesis: a systematic review and metaanalysis. Spine J 2017;17:1712-21.

21. Lara-Almunia M, Gomez-Moreta JA, HernandezVicente J. Posterior lumbar interbody fusion with instrumented posterolateral fusion in adult spondylolisthesis: description and association of clinicosurgical variables with prognosis in a series of 36 cases. Int J Spine Surg 2015;9:22.

22. Hsieh CS, Lee HC, Oh HS, Park SJ, Hwang BW, Lee $\mathrm{SH}$. Anterior lumbar interbody fusion with percutaneous pedicle screw fixation for multiple-level isthmic spondylolisthesis. Clin Neurol Neurosurg 2017;158:49-52.

23. Lee GW, Son JH, Ahn MW, Kim HJ, Yeom JS. The comparison of pedicle screw and cortical screw in posterior lumbar interbody fusion: a prospective randomized noninferiority trial. Spine J 2015;15:151926.

24. Pinson H, Hallaert G, Herregodts P, et al. Outcome of anterior lumbar interbody fusion: a retrospective study of clinical and radiologic parameters. World Neurosurg 2017;103:772-9.

25. Hayashi K, Matsumura A, Konishi S, Kato M, Namikawa T, Nakamura H. Clinical outcomes of posterior lumbar interbody fusion for patients 80 years of age and older with lumbar degenerative disease: minimum 2 years' follow-up. Global Spine J 2016;6:66572.

26. Kim JS, Lee KY, Lee SH, Lee HY. Which lumbar interbody fusion technique is better in terms of level for the treatment of unstable isthmic spondylolisthesis? J Neurosurg Spine 2010;12:171-7.

27. Okuda S, Oda T, Yamasaki R, Haku T, Maeno T, Iwasaki M. Posterior lumbar interbody fusion with total facetectomy for low-dysplastic isthmic spondylolisthesis: effects of slip reduction on surgical outcomes: clinical article. J Neurosurg Spine 2014;21:171-8.

28. Gologorsky Y, Skovrlj B, Steinberger J, et al. Increased incidence of pseudarthrosis after unilateral instrumented transforaminal lumbar interbody fusion in patients with lumbar spondylosis: clinical article. J Neurosurg Spine 2014;21:601-7.

29. Sakaura H, Miwa T, Yamashita T, Kuroda Y, Ohwada T. Posterior lumbar interbody fusion with cortical bone trajectory screw fixation versus posterior lumbar interbody fusion using traditional pedicle screw fixation for degenerative lumbar spondylolisthesis: a comparative study. J Neurosurg Spine 2016;25:591-5.

30. Fleege C, Arabmotlagh M, Rother W, Rauschmann M, Rickert M. ALIF and PLIF interposition in lowgrade isthmic spondylolisthesis L5/S1: longtermcomparison of interbody fusion techniques (ALIF PLIF). Orthopade 2016;45:760-9.

31. Baeesa SS, Medrano BG, Noriega DC. Long-term outcomes of posterior lumbar interbody fusion using stand-alone ray threaded cage for degenerative disk disease: a 20-year follow-up. Asian Spine J 2016;10:1100-5.

32. Malham GM, Parker RM, Blecher CM, Chow FY, Seex KA. Choice of approach does not affect clinical and radiologic outcomes: a comparative cohort of patients having anterior lumbar interbody fusion and patients having lateral lumbar interbody fusion at 24 months. Global Spine J 2016;6:472-81.

33. Hosono N, Namekata M, Makino T, et al. Perioperative complications of primary posterior lumbar inter- 
body fusion for nonisthmic spondylolisthesis: analysis of risk factors. J Neurosurg Spine 2008;9:403-7.

34. Min JH, Jang JS, Lee SH. Comparison of anteriorand posterior-approach instrumented lumbar interbody fusion for spondylolisthesis. J Neurosurg Spine 2007;7:21-6.

35. Quraishi NA, Konig M, Booker SJ, et al. Access related complications in anterior lumbar surgery performed by spinal surgeons. Eur Spine J 2013;22(Suppl 1):S16-20.

36. Rao PJ, Loganathan A, Yeung V, Mobbs RJ. Outcomes of anterior lumbar interbody fusion surgery based on indication: a prospective study. Neurosurgery 2015;76:7-24.

37. Li H, Wang H, Zhu Y, Ding W, Wang Q. Incidence and risk factors of posterior cage migration following decompression and instrumented fusion for degenerative lumbar disorders. Medicine (Baltimore) 2017;96:e7804.

38. Teng I, Han J, Phan K, Mobbs R. A meta-analysis comparing ALIF, PLIF, TLIF and LLIF. J Clin Neurosci 2017;44:11-7.

39. Mobbs RJ, Loganathan A, Yeung V, Rao PJ. Indications for anterior lumbar interbody fusion. Orthop Surg 2013;5:153-63.

40. Memtsoudis SG, Vougioukas VI, Ma Y, Gaber-Baylis LK, Girardi FP. Perioperative morbidity and mortality after anterior, posterior, and anterior/posterior spine fusion surgery. Spine (Phila Pa 1976) 2011;36:186777.
41. Mobbs RJ, Phan K, Daly D, Rao PJ, Lennox A. Approach-related complications of anterior lumbar interbody fusion: results of a combined spine and vascular surgical team. Global Spine J 2016;6:147-54.

42. Paik NC. Incisional hernia after anterior lumbar interbody fusion. Spine J 2010;10:844.

43. Villavicencio AT, Burneikiene S, Bulsara KR, Thramann JJ. Perioperative complications in transforaminal lumbar interbody fusion versus anterior-posterior reconstruction for lumbar disc degeneration and instability. J Spinal Disord Tech 2006;19:92-7.

44. Cole CD, McCall TD, Schmidt MH, Dailey AT. Comparison of low back fusion techniques: transforaminal lumbar interbody fusion (TLIF) or posterior lumbar interbody fusion (PLIF) approaches. Curr Rev Musculoskelet Med 2009;2:118-26.

45. Mahesh B, Upendra B, Vijay S, Kumar GA, Reddy S. Complication rate during multilevel lumbar fusion in patients above 60 years. Indian J Orthop 2017;51:13946.

46. Abbushi A, Cabraja M, Thomale UW, Woiciechowsky C, Kroppenstedt SN. The influence of cage positioning and cage type on cage migration and fusion rates in patients with monosegmental posterior lumbar interbody fusion and posterior fixation. Eur Spine J 2009;18:1621-8. 